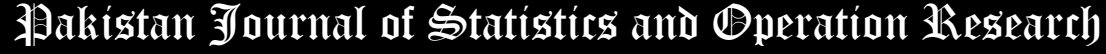

\section{Transmuted Topp-Leone Weibull Lifetime Distribution: Statistical Properties and Different Method of Estimation}

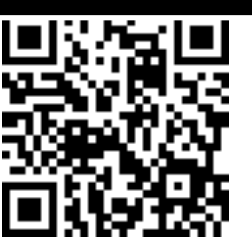

\author{
Mohamed Ibrahim ${ }^{1}$ and Haitham M. Yousof ${ }^{2 *}$
}

* Corresponding Author

1. Department of Applied Statistics and Insurance, Faculty of Commerce, Damietta University, Egypt. mohamed_ibrahim@du.edu.eg

2. Department of Statistics, Mathematics and Insurance, Benha University, Egypt, haitham.yousof@fcom.bu.edu.eg

\begin{abstract}
In this work we focus on proposing a new lifetime Weibull type model called the transmuted Topp-Leone Weibull and studying its properties. We derive some new bivariate and multivariate transmuted Topp-Leone Weibull versions using "Farlie Gumbel Morgenstern (FGM) Copula", "modified FGM Copula", "Clayton Copula" and "Renyi's entropy Copula". The estimation of its unknown parameters is carried out by considering different method of estimation. The statistical performances of all methods are studied by two real data sets and a numerical Monte Carlo simulation. The Cramer-Von Mises method is the best method for modeling the carbon fibers data. The maximum likelihood method is the best method for modeling the Leukemia data, however all other methods performed well.
\end{abstract}

Key Words: Weibull Distribution, Farlie Gumbel Morgenstern; Clayton Copula; Least Square; Topp-Leone; Likelihood Estimation; Weighted Least Square; Cramer-Von-Mises; Bootstrapping.

Mathematical Subject Classification: 62N01; 62N02; 62E10.

\section{Introduction and genesis}

Consider the two-parameters Weibull (W) type lifetime model with probability density function (P.D.F) and cumulative function (C.D.F) are given (for $w \geq 0$ ) as

$$
g_{a, b}(w)=b a^{b} x^{b-1} e^{-(a w)^{b}}
$$

and

$$
G_{a, b}(w)=1-e^{-(a w)^{b}}
$$

Where parameter $a>0$ dominate the shape of the model however $b>0$ is a scale parameter. Clearly, when $a=0$ we have the one parameter Weibull model. In this paper, we study a new model called the transmuted Topp-LeoneW (TTL-W) model. Based on the TTL-G family, we construct the new four-parameter TTL-W model. Let $g_{\underline{\varphi}}(w)=$ $\frac{d}{d w} G_{\underline{\boldsymbol{\varphi}}}(w)$ and $G_{\underline{\boldsymbol{\varphi}}}(w)$ denote the P.D.F and the C.D.F of the baseline model with parameter vector $\underline{\boldsymbol{\varphi}}=(a, b)$ in our case. Using the transmuted G (T-G) family pioneered by Shaw and Buckley (2007) and the Topp-Leone G (TL-G) family pioneered by Rezaei et al. (2016), Yousof et al. (2017b) defined the C.D.F of their transmuted Topp-Leone G (TTL-G) family by

$$
F_{\lambda, \alpha, \underline{\varphi}}(w)=-\lambda\left\{1-\left[-G_{\underline{\varphi}}(w)+1\right]^{2}\right\}^{2 \alpha}+(1+\lambda)\left\{1-\left[-G_{\underline{\varphi}}(w)+1\right]^{2}\right\}^{\alpha}
$$

The P.D.F of the TTL-G family is given by 


\section{Theorem 1:}

$$
f_{\lambda, \alpha, \underline{\varphi}}(w)=2 \alpha g_{\underline{\varphi}}(w)\left[-G_{\underline{\varphi}}(w)+1\right] \frac{1-2 \lambda\left\{1-\left[-G_{\underline{\varphi}}(w)\right]^{2}+1\right\}^{\alpha}+\lambda}{\left\{-\left[1-G_{\underline{\varphi}}(w)+1\right]^{2}\right\}^{1-\alpha}} .
$$

Let $T$ be a random variable (rv) having the well-known Exponentiated W (Exp-W) distribution with positive parameters $a, b$ and $\boldsymbol{\delta}$. Then the P.D.F and C.D.F of $T$ are given by $\boldsymbol{\pi}_{\boldsymbol{\delta}, a, b}(t)=\boldsymbol{\delta} b a^{b} t^{b-1}\left[-e^{-(a t)^{b}}+1\right]^{\boldsymbol{\delta}-1}$ $e^{-(a t)^{b}}$ and $\boldsymbol{\Pi}_{\boldsymbol{\delta}, a, b}(t)=\left[-e^{-(a t)^{b}}+1\right]^{\boldsymbol{\delta}}$ where $\boldsymbol{\pi}_{\delta, a, b}(t)=\frac{d}{d t} \boldsymbol{\Pi}_{\boldsymbol{\delta}, a, b}(t)$. The $q$ th ordinary $\left(\mu_{q}^{\prime}\right)$ and incomplete moment $\left(\boldsymbol{\Phi}_{q}(t)\right)$ of $T$ are given by

and

$$
\left.\mu_{q}^{\prime}\right|_{[q>-b]}=\Gamma\left(1+\frac{q}{b}\right) \sum_{\boldsymbol{\tau}=0}^{\infty} a^{-q} \boldsymbol{\delta}(-1)^{\boldsymbol{\tau}}(\boldsymbol{\tau}+1)^{-(q+b) / b}\left(\begin{array}{c}
\boldsymbol{\delta}-1 \\
\boldsymbol{\tau}
\end{array}\right),
$$

$$
\left.\boldsymbol{\phi}_{q}(t)\right|_{[q>-b]}=\boldsymbol{\gamma}\left(1+\frac{q}{b},(a t)^{b}\right) \sum_{\boldsymbol{\tau}=0}^{\infty} a^{-q} \boldsymbol{\delta}(-1)^{\boldsymbol{\tau}}(\boldsymbol{\tau}+1)^{-(q+b) / b}\left(\begin{array}{c}
\boldsymbol{\delta}-1 \\
\boldsymbol{\tau}
\end{array}\right),
$$

Respectively, where $\gamma(v, q)$ is the incomplete gamma function

$$
\left.\gamma(V, \mathbf{c})\right|_{(V \neq 0,-1,-2, \ldots)}=\int_{0}^{\mathbf{c}} t^{V-1} \exp (-t) d t=\frac{\mathbf{c}^{v}}{V}\left\{1 F_{1}[V ; V \mathbf{c}+1 ;-\mathbf{c}]\right\}=\sum_{\kappa=0}^{\infty} \frac{(-1)^{\kappa}}{\kappa !(V+\kappa)} \mathbf{c}^{V+\kappa},
$$

the function $1 F_{1}[\because, \cdot]$ is a called the confluent hypergeometric function $\left.\Gamma(V, \mathbf{c})\right|_{(t>0)}=\int_{\mathbf{c}}^{\infty} t^{V-1} \exp (-t) d t$ and $\boldsymbol{\Gamma}(V, \mathbf{c})+\gamma(V, \mathbf{c})=\boldsymbol{\Gamma}(V)$. Using (2) and (3) we get the C.D.F of the TTL-W model as

$$
F_{\underline{\Psi}}(w)=(1+\lambda)\left[-e^{-2(a w)^{b}}+1\right]^{\alpha}-\lambda\left[-e^{-2(a w)^{b}}+1\right]^{2 \alpha}
$$

where $\underline{\Psi}=(\lambda, \alpha, a, b)$. The corresponding P.D.F is obtained by

$$
f_{\underline{\Psi}}(w)=2 \alpha b a^{b} w^{b-1} e^{-2(a w)^{b}} \frac{\left\{-2 \lambda\left[1-e^{-2(a w)^{b}}\right]^{\alpha}+\lambda+1\right\}}{\left[-e^{-2(a w)^{b}}+1\right]^{1-\alpha}} .
$$

Table 1 gives some sub models of the TTL-W model. As illustrated in Table 1, the new model generalizes fifteen sub models. The hazard rate function (H.R.F) of the TTL-W model can calculated from $f_{\Psi}(w) /\left[1-F_{\underline{\Psi}}(w)\right]$. Figure 1 provides some plots of the P.D.F and H.R.F of the TTL-W model to show its flexibility. The P.D.F can be unimodal and right skewed. The H.R.F can be "upside down", "monotone-decreasing", "bathtub (U-shaped)", "monotoneincreasing", "J shaped" and "constant" shapes.
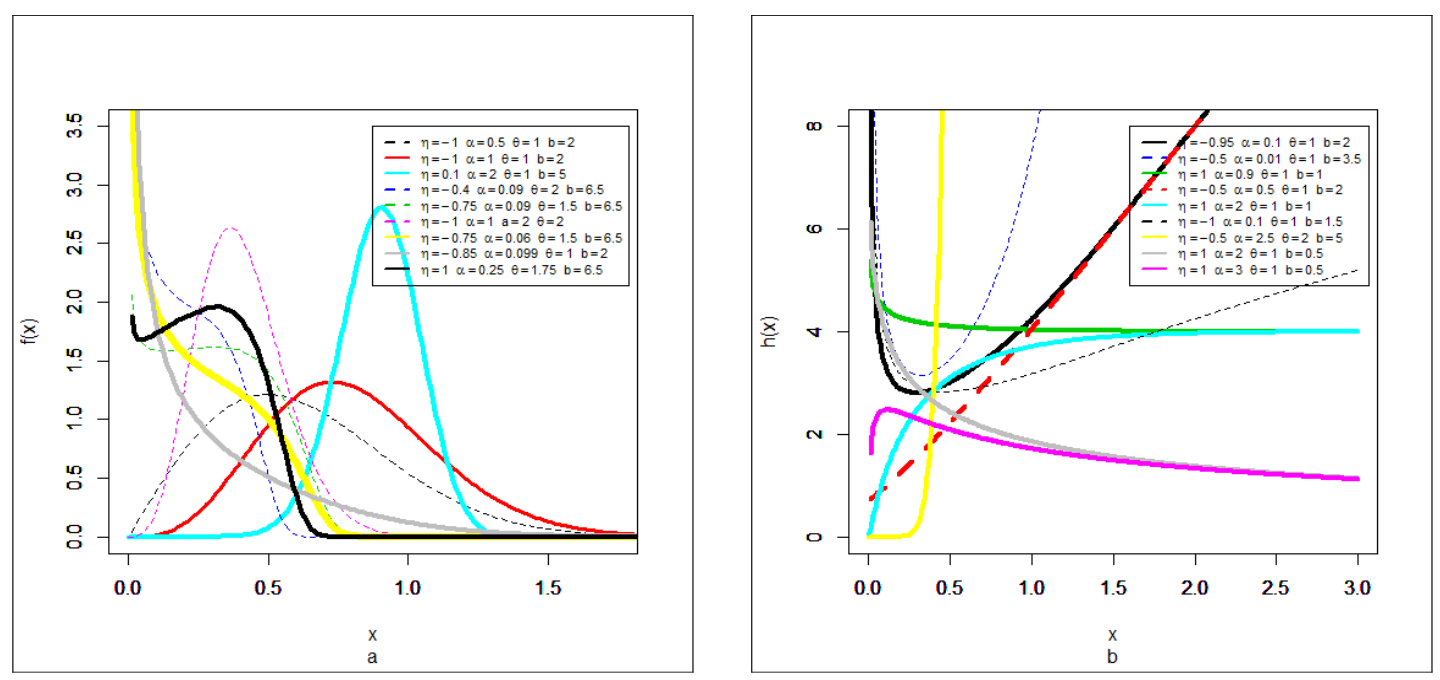

Figure 1: Plots of the TTL-W P.D.F and H.R.F for selected parameter values. 
Table 1: Sub models of the TTL-W model.

\begin{tabular}{cccccc}
\hline & $\lambda$ & $\alpha$ & $a$ & $b$ & Reduced model \\
\hline 1 & 0 & $\alpha$ & $a$ & $b$ & ExpTL-W \\
2 & 0 & $\alpha$ & $a$ & 2 & ExpTL-Rayleigh \\
3 & 0 & $\alpha$ & $a$ & 1 & ExpTL-exponential \\
4 & 0 & 1 & $a$ & $b$ & TL-W \\
5 & 0 & 1 & $a$ & 2 & TL-Rayleigh \\
6 & 0 & 1 & $a$ & 1 & TL-exponential \\
7 & 0 & 1 & 1 & $b$ & TL-W \\
8 & 0 & 1 & 1 & 2 & TL-Rayleigh \\
9 & 0 & 1 & 1 & 1 & TL-exponential \\
10 & $\lambda$ & 1 & $a$ & $b$ & T-W \\
11 & $\lambda$ & 1 & $a$ & 2 & T-Rayleigh \\
12 & $\lambda$ & 1 & $a$ & 1 & T-exponential \\
13 & $\lambda$ & 1 & 1 & $b$ & T-W \\
14 & $\lambda$ & 1 & 1 & 2 & T- Rayleigh \\
15 & $\lambda$ & 1 & 1 & 1 & T-exponential \\
\hline
\end{tabular}

The C.D.F of the TTL-W model in (5) can be expressed as

$$
F_{\underline{\mathbf{\Psi}}}(w)=(1+\lambda) \sum_{\boldsymbol{\tau}=0}^{\infty}(-1)^{\boldsymbol{\tau}}\left(\begin{array}{l}
\alpha \\
\boldsymbol{\tau}
\end{array}\right) e^{-2 \boldsymbol{\tau}(a w)^{b}}-\lambda \sum_{\boldsymbol{\tau}=0}^{\infty}(-1)^{\boldsymbol{\tau}}\left(\begin{array}{l}
\alpha \\
\boldsymbol{\tau}
\end{array}\right) e^{-2 \boldsymbol{\tau}(a w)^{b}}
$$

which can be rewritten as

$$
F_{\underline{\Psi}}(w)=(1+\lambda) \sum_{\boldsymbol{\tau}=0}^{\infty} \sum_{k=0}^{2 \boldsymbol{\tau}}(-1)^{\boldsymbol{\tau}+k} e^{-k(a x w}\left(\begin{array}{c}
\alpha \\
\boldsymbol{\tau}
\end{array}\right)\left(\begin{array}{c}
2 \boldsymbol{\tau} \\
k
\end{array}\right)-\lambda \sum_{\boldsymbol{\tau}=0}^{\infty} \sum_{k=0}^{2 \boldsymbol{\tau}}(-1)^{\boldsymbol{\tau}+k} e^{-k(a w)^{b}}\left(\begin{array}{c}
2 \alpha \\
\boldsymbol{\tau}
\end{array}\right)\left(\begin{array}{c}
2 \boldsymbol{\tau} \\
k
\end{array}\right) .
$$

Then, after some algebra we have $F_{\underline{\Psi}}(w)=\sum_{\tau=0}^{\infty} \sum_{k=0}^{2 \tau} \xi_{h, k} e^{-k(a w)^{b}}$, then

$$
F_{\underline{\Psi}}(w)=\sum_{\tau=0}^{\infty} \sum_{k=0}^{2 \tau} \xi_{\boldsymbol{\tau}, k} \Pi_{k}(w)
$$

where $\boldsymbol{\xi}_{\boldsymbol{\tau}, k}=(-1)^{\boldsymbol{\tau}+k}\left[\left(\begin{array}{c}\alpha \\ \boldsymbol{\tau}\end{array}\right)+\lambda\left(\begin{array}{c}\alpha \\ \boldsymbol{\tau}\end{array}\right)-\lambda\left(\begin{array}{c}2 \alpha \\ \boldsymbol{\tau}\end{array}\right)\right]\left(\begin{array}{c}2 \boldsymbol{\tau} \\ k\end{array}\right)$, and $\boldsymbol{\Pi}_{\boldsymbol{\delta}}(w)=\left[G_{a, b}(w)\right]^{\boldsymbol{\delta}}$ is the C.D.F of the Exp-W distribution with power parameter $\boldsymbol{\delta}$. Then

$$
f_{\underline{\Psi}}(w)=\sum_{\tau=0}^{\infty} \sum_{k=0}^{2 \tau} \xi_{\boldsymbol{\tau}, k} \pi_{k}(w)
$$

where $\boldsymbol{\pi}_{\boldsymbol{\delta}}(w)=\boldsymbol{\delta} g_{a, b}(w)\left[G_{a, b}(w)\right]^{\boldsymbol{\delta}-1}$ is the P.D.F of the Exp-W distribution with power parameter $\boldsymbol{\delta}$. Some useful extension of the Weibull model is recently developed by Yousof. etal., (2015) (transmuted exponentiated generalized W.), Aryal. etal., (2017) (Topp Leone generated W.), Aryal. and Yousof. (2017) (exponentiated. generalized. W. Poisson), Merovci. Et al., (2017) (exponentiated transmuted W.), Yousof. et al., (2017a) (new four-parameter W.), Brito et al., (2017) (Topp-Leone Odd Log-Logistic W.), Hamedani. et al., (2018) (Type I general exponential W.), Alizadeh. etal. (2017) (generalized. odd generalized. exponential W.), Almamy. et al. (2018) (odd Lindley W.), Cordeiro. et al., (2018) (Burr XII W.), Yousof. et al., (2018) (Burr-Hatke W.), Korkmaz et al., (2019) (Odd Power Lindley W.), among others.

\section{Simple type Copula}

\subsection{Bivariate TTL-W (BvTTL-W) type using FGM Copula}

Consider the joint C.D.F (J.C.D.F) of the FGM family (Gumbel (1960), Morgenstern (1956), Farlie, D. J. G. (1960), Gumbel (1961), Johnson and Kotz (1977) and Johnson and $\operatorname{Kotz}(1975)$ ), then $C_{\mathcal{B}}(\boldsymbol{q}, \boldsymbol{p})=\boldsymbol{q} \boldsymbol{p}(1+\mathcal{B} \overline{\boldsymbol{q} p})$, where the continuous marginal function $\boldsymbol{q} \in[0,1], \boldsymbol{p} \in[0,1], \mathcal{B} \in[-1,1]$ is a dependence parameter and for every $C_{\mathcal{B}}(\boldsymbol{q}, 0)=$ 
$C_{\mathcal{B}}(0, \boldsymbol{p})=\left.0\right|_{(\boldsymbol{q}, \boldsymbol{p} \in(0,1))}$ "grounded minimum" and $C_{\mathcal{B}}(\boldsymbol{q}, 1)=\boldsymbol{q}$ and $C_{\mathcal{B}}(1, \boldsymbol{p})=\boldsymbol{p}$ "grounded maximum". Then, setting $\overline{\boldsymbol{q}}=\left.\overline{\boldsymbol{q}}_{\underline{\boldsymbol{\Psi}}_{1}}\right|_{\underline{\boldsymbol{\Psi}}_{1}>0}$, and $\overline{\boldsymbol{p}}=\left.\overline{\boldsymbol{p}}_{\underline{\boldsymbol{\Psi}}_{2}}\right|_{\boldsymbol{\Psi}_{2}>0}$. Where $C_{\mathcal{B}}(\boldsymbol{q}, \boldsymbol{p})=F\left(y_{1}, y_{2}\right)=C\left(F_{\boldsymbol{\Psi}_{1}}\left(y_{1}\right), F_{\underline{\Psi}_{2}}\left(y_{2}\right)\right)$. The J.P.D.F can be derived from $c_{\mathcal{B}}(\boldsymbol{q}, \boldsymbol{p})=1+\left.\mathcal{B} \boldsymbol{q}^{*} \boldsymbol{p}^{*}\right|_{\left(\boldsymbol{q}^{*}=1-2 \boldsymbol{q} \text { and } \boldsymbol{p}^{*}=1-2 \boldsymbol{p}\right)}$ or from $f\left(y_{1}, y_{2}\right)=f_{\underline{\boldsymbol{\Psi}}_{1}}\left(y_{1}\right) f_{\underline{\Psi}_{2}}\left(y_{2}\right) c\left(F_{\underline{\Psi}_{1}}\left(y_{1}\right), F_{\underline{\Psi}_{2}}\left(y_{2}\right)\right)$.

\subsection{BvTTL-W type via modified FGM Copula}

Consider the following modified FGM copula defined as (Rodriguez-Lallena and Ubeda-Flores (2004)) where

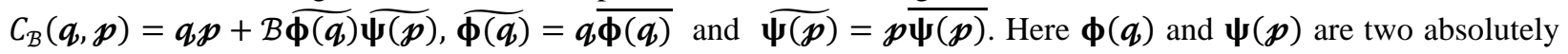
continuous functions on $(0,1)$ where $\boldsymbol{\phi}(0)=\boldsymbol{\phi}(1)=\boldsymbol{\psi}(0)=\boldsymbol{\psi}(1)=0$. Let

$$
\begin{aligned}
& a=\inf \left\{\frac{\partial}{\partial \boldsymbol{q}} \widetilde{\boldsymbol{\phi ( q )}}: \mathcal{H}_{1}\right\}<0, b=\sup \left\{\frac{\partial}{\partial \boldsymbol{q}} \widetilde{\boldsymbol{\phi ( q )}}: \mathcal{H}_{1}\right\}<0, \\
& c=\inf \left\{\frac{\partial}{\partial \boldsymbol{p}} \widetilde{\boldsymbol{\Psi}(\boldsymbol{p})}: \mathcal{H}_{2}\right\}>0, d=\sup \left\{\frac{\partial}{\partial \boldsymbol{p}} \widetilde{\boldsymbol{\Psi}(\boldsymbol{p})}: \mathcal{H}_{2}\right\}>0 .
\end{aligned}
$$

Then $1 \leq \min (a b, c d)$ where

$$
\begin{gathered}
\mathcal{q} \frac{\partial}{\partial \boldsymbol{q}} \boldsymbol{\phi}(\boldsymbol{q})=\frac{\partial}{\partial \boldsymbol{q}} \widetilde{\boldsymbol{\phi ( q )}}-\boldsymbol{\phi}(\boldsymbol{q}), \\
\mathcal{H}_{1}=\left\{\boldsymbol{q} \in(0,1): \frac{\partial}{\partial \boldsymbol{q}} \widetilde{\boldsymbol{\phi ( q )}} \quad \text { exists }\right\},
\end{gathered}
$$

and

$$
\mathcal{H}_{2}=\left\{\boldsymbol{p} \in(0,1): \frac{\partial}{\partial \boldsymbol{p}} \widetilde{\boldsymbol{\Psi ( p )}} \text { exists }\right\}
$$

\subsubsection{BvTTL-W-FGM (Type-I) model}

Here, we consider the following functional form for both $\boldsymbol{\phi}(\boldsymbol{q})$ and $\boldsymbol{\psi}(\boldsymbol{p})$ where $\overline{\boldsymbol{\phi}(\boldsymbol{q})}=\left.\boldsymbol{q}\left[1-F_{\underline{\Psi}_{1}}(\boldsymbol{q})\right]\right|_{\underline{\Psi}_{1}>0}$, and $\widetilde{\boldsymbol{\Psi}(\boldsymbol{p})}=\left.\boldsymbol{p}\left[1-F_{\underline{\Psi}_{2}}(\boldsymbol{p})\right]\right|_{\underline{\Psi}_{2}>0}$. Then using $C_{\mathcal{B}}(\boldsymbol{q}, \boldsymbol{p})=\boldsymbol{q} \boldsymbol{p}+\mathcal{B} \widetilde{\boldsymbol{\phi}(\boldsymbol{q})} \widetilde{\boldsymbol{\Psi}(\boldsymbol{p})}$, the BvTTL-W-FGM (Type-I) can be derived.

\subsubsection{BvTTL-W-FGM (Type-II) model:}

Consider the following functional form for both $\phi(q)$ and $\boldsymbol{\psi}(\boldsymbol{p})$ which satisfy all the conditions stated earlier where

$$
\left.\boldsymbol{\phi}(\boldsymbol{q})\right|_{\left(\mathcal{B}_{1}>0\right)}=\boldsymbol{q}^{\mathcal{B}_{1}}(1-\boldsymbol{q})^{1-\mathcal{B}_{1}} \text { and }\left.\boldsymbol{\psi}(\boldsymbol{p})\right|_{\left(\mathcal{B}_{2}>0\right)}=\boldsymbol{p}^{\mathcal{B}_{2}}(1-\boldsymbol{p})^{1-\mathcal{B}_{2}}
$$

The corresponding bivariate copula (henceforth, BvTTL-W-FGM (Type-II) copula) can be derived from

$$
C_{\mathcal{B}, B_{1}, B_{2}}(\boldsymbol{q}, \boldsymbol{p})=\boldsymbol{q} \boldsymbol{p}\left[1+\mathcal{B} \boldsymbol{q}^{\mathcal{B}_{1}} \boldsymbol{p}^{\mathcal{B}_{2}}(1-\boldsymbol{q})^{1-\mathcal{B}_{1}}(1-\boldsymbol{p})^{1-\mathcal{B}_{2}}\right] .
$$

\subsubsection{BvTTL-W-FGM (Type-III) model:}

Consider the following functional form for both $\boldsymbol{\phi}(\boldsymbol{q})$ and $\boldsymbol{\psi}(\boldsymbol{q})$ which satisfy all the conditions stated earlier where $\boldsymbol{\phi}(\boldsymbol{q})=\boldsymbol{q}[\log (1+\overline{\boldsymbol{q}})]$ and $\boldsymbol{\psi}(\boldsymbol{p})=\boldsymbol{p}[\log (1+\bar{p})]$. In this case, one can also derive a closed form expression for the associated C.D.F of the BvTTL-W-FGM (Type-III).

\subsubsection{BvTTL-W-FGM (Type-IV) model:}

due to Ghosh and Ray (2016) the C.D.F of the BvTTL-W-FGM (Type-IV) model can be derived from $C(\boldsymbol{q}, \boldsymbol{p})=$ $\boldsymbol{q} F^{-1}(\boldsymbol{p})+\boldsymbol{p} F^{-1}(\boldsymbol{q})-F^{-1}(\boldsymbol{q}) F^{-1}(\boldsymbol{p})$ where $F^{-1}(\boldsymbol{q})$ and $F^{-1}(\boldsymbol{p})$ are derived before.

\subsection{BvTTL-W type via Clayton Copula}

The Clayton Copula can be considered as $C\left(\boldsymbol{q}_{1}, \boldsymbol{q}_{2}\right)=\left.\left(\boldsymbol{q}_{1}^{-\mathcal{B}}+\boldsymbol{q}_{2}^{-\mathcal{B}}-1\right)^{-\frac{1}{\mathcal{B}}}\right|_{\mathcal{B} \in[0, \infty]}$. Let us assume that $Z \sim$ TTL-W $\left(\underline{\Psi}_{1}\right)$ and $T \sim$ TTL-W $\left(\underline{\boldsymbol{\Psi}}_{2}\right)$. Then, setting $\boldsymbol{q}_{1}=\left.\boldsymbol{q}(z)\right|_{\boldsymbol{\Psi}_{1}>0}$ and $\boldsymbol{q}_{2}=\left.\boldsymbol{q}(t)\right|_{\underline{\Psi}_{2}>0}$. Then, the BvTTL-W type distribution can be derived from $F(z, t)=C\left(F_{\underline{\Psi}_{1}}(z), F_{\underline{\Psi}_{2}}(t)\right)$.

\subsection{BvTTL-W type via Renyi's entropy}

Consider theorem of Pougaza and Djafari (2011) where $C(\boldsymbol{q}, \boldsymbol{p})=z_{2} \boldsymbol{q}+z_{1} \boldsymbol{p}-z_{1} z_{2}$. The associated BvTTL-W will be $\left.C\left(z_{1}, z_{2}\right)\right|_{\left(\alpha=\alpha_{1}=\alpha_{2}\right)}=C\left(F_{\underline{\Psi}_{1}}\left(z_{1}\right), F_{\underline{\Psi}_{2}}\left(z_{1}\right)\right)$. The $M$-dimensional extension can be derived from $C\left(\boldsymbol{q}_{i}\right)=$ $\left[\sum_{i=1}^{M} \boldsymbol{q}_{i}^{-\mathcal{B}}+1-M\right]^{-\frac{1}{\mathcal{B}}}$. 


\section{Statistical properties}

\subsection{Moments}

The $M^{t h}$ ordinary moment of $W$ is given by $\mu_{M}^{\prime}=\mathbf{E}\left(W^{M}\right)=\int_{-\infty}^{\infty} w^{M} f_{\mathbf{\Psi}}(w) d w$. Then, using Theorem 1 we obtain

$$
\left.\mu_{M}^{\prime}\right|_{[M>-b]}=\Gamma\left(1+\frac{M}{b}\right) \sum_{m, \tau=0}^{\infty} \sum_{k=0}^{2 \tau} \xi_{m, \tau}^{(M, k)},
$$

where $\xi_{m, \mathbf{\tau}, k}^{(M, k)}=\boldsymbol{\xi}_{\mathbf{\tau}, k} k(-1)^{m} a^{-M}(m+1)^{-(M+b) / b}\left(\begin{array}{c}-1+k \\ m\end{array}\right)$. Setting $M=1$ in (10), we have the mean of $X$ as $\left.\mu_{1}^{\prime}\right|_{[M>-b]}=\Gamma\left(1+\frac{1}{b}\right) \sum_{m, \mathbf{\tau}=0}^{\infty} \sum_{k=0}^{2 \tau} \xi_{m, \mathbf{\tau}, k}^{(1, k)}$ where $\boldsymbol{\xi}_{m, \boldsymbol{\tau}, k}^{(1, k)}=\boldsymbol{\xi}_{\boldsymbol{\tau}, k} k(-1)^{m} a^{-1}(m+1)^{-(1+b) / b}\left(\begin{array}{c}k-1 \\ m\end{array}\right)$. The moment generating function (MGF) can be derived from equation (9) and Theorem 1 as

$$
\left.M_{W}(t)\right|_{[r>-b]}=\Gamma\left(1+\frac{r}{b}\right) \sum_{m, r, \boldsymbol{\tau}=0}^{\infty} \sum_{k=0}^{2 \tau} \mathbf{Q}_{m, \tau, h, k}^{(r, k)},
$$

where $\mathbf{Q}_{m, r, \boldsymbol{\tau}, k}^{(r, k)}=\boldsymbol{\xi}_{\boldsymbol{\tau}, k} k(-1)^{m} t^{r} \boldsymbol{\Gamma}^{-1}(1+r) a^{-r}(m+1)^{-(r+b) / b}\left(\begin{array}{c}-1+k \\ m\end{array}\right)$. The skewness $(\operatorname{sk}(W))$ and kurtosis $(\mathrm{ku}(W))$ measures can be calculated from the ordinary moments using well-known relationships (see Table 2). The mean $\left(\mu_{1}^{\prime}\right)$, variance $(V(W)), \operatorname{sk}(W)$ and $\mathrm{ku}(W)$ of the proposed distribution are computed numerically for some selected values of parameter $\lambda, \alpha, a$ and $b$ using the $\mathrm{R}$ software. The $\mathrm{sk}(W)$ of the proposed distribution can range in the interval $(-0.68,5.1)$ and this means that the new model can be left and right skewed, whereas the $\mathrm{ku}(\mathrm{X})$ of the proposed distribution varies in the interval $(-398.1,54.1)$.

Table 2: $\mu_{1}{ }^{\prime}, \mathrm{V}(\mathrm{X}), \mathrm{sk}(\mathrm{X})$ and $\mathrm{ku}(\mathrm{X})$ of the proposed distribution.

\begin{tabular}{llll|llll}
\hline$\lambda$ & $\alpha$ & $\mathrm{a}$ & $\mathrm{b}$ & $\mu^{\prime}{ }^{\prime}$ & $\mathrm{V}(\mathrm{W})$ & $\mathrm{sk}(\mathrm{W})$ & $\mathrm{ku}(\mathrm{W})$ \\
\hline 1.5 & 1 & 0.5 & 0.5 & 1.750000 & 8.562500 & 5.119910 & 54.08711 \\
& & & 1 & 1.500000 & 1.250000 & 1.609969 & 7.080000 \\
& & & 5 & 1.805566 & 0.080897 & -0.167734 & 3.000812 \\
& & & 10 & 1.894169 & 0.023256 & -0.425684 & 3.341781 \\
& & & 20 & 1.944749 & 0.006287 & -0.566375 & 3.638961 \\
& & & 75 & 1.977314 & 0.001057 & -0.655667 & 3.870117 \\
& & & 1.984787 & 0.000475 & -0.675816 & 3.903296 \\
\hline 5 & 3 & 0.1 & 3 & 10.42449 & 2.905149 & 0.2661283 & 3.117302 \\
& & 0.5 & & 2.084897 & 0.116206 & 0.2661283 & 3.117302 \\
& 2 & & 0.521224 & 0.007263 & 0.2661268 & 3.117339 \\
& & 5 & & 0.208490 & 0.001162 & 0.2661283 & 3.117302 \\
& & 10 & & 0.104245 & 0.000290 & 0.2661283 & 3.117434 \\
& 20 & & 0.052122 & $7.2628 \times 10^{5}$ & 0.2661245 & -398.058 \\
\hline 10 & 1 & 5 & 5 & 0.180557 & 0.00080897 & -0.167733 & 3.000813 \\
& 10 & & & 0.222872 & 0.00022656 & 0.2859269 & 3.150747 \\
& 20 & & & 0.231334 & 0.00017336 & 0.3825359 & 3.251737 \\
& 50 & & & 0.240914 & 0.00012849 & 0.4839039 & 3.394393 \\
\hline
\end{tabular}

\subsection{Incomplete moments}

The $M$ th incomplete moment can be expressed as $\boldsymbol{\phi}_{M}(\mathbf{z})=\int_{-\infty}^{\mathbf{z}} w^{M} f_{\mathbf{\Psi}}(w) d x$. Then, using Theorem 1 we have

$$
\left.\boldsymbol{\phi}_{M}(\mathbf{z})\right|_{[M>-b]}=\gamma\left(1+\frac{M}{b},(a \mathbf{z})^{b}\right) \sum_{m, \tau=0}^{\infty} \sum_{k=0}^{2 \tau} \phi_{m, \tau, k}^{(M, k)},
$$


where $\emptyset_{m, \mathbf{\tau}, k}^{(M, k)}=\xi_{\tau, k} k(-1)^{m} a^{-M}(m+1)^{-(M+b) / b}\left(\begin{array}{c}-1+k \\ m\end{array}\right)$. A general equation for $\boldsymbol{\phi}_{1}(t)$ can be derived from (11) as $\quad \boldsymbol{\phi}_{1}(\mathbf{z}) \mid=\boldsymbol{\gamma}\left(1+\frac{1}{b},(a \mathbf{z})^{b}\right) \sum_{m, \mathbf{\tau}=0}^{\infty} \sum_{k=0}^{2 \boldsymbol{\tau}} \emptyset_{m, \mathbf{\tau}, k}^{(1, k)}$ where $\emptyset_{m, \mathbf{\tau}, k}^{(1, k)}=\xi_{h, k} k(-1)^{m} a^{-1}(m+1)^{-(1+b) / b}\left(\begin{array}{c}-1+k \\ m\end{array}\right)$ and $\boldsymbol{\phi}_{1}(z)$ is the first incomplete moment of the new distribution.

3.3 Probability weighted moments (PWMs)

The $(M, \mathbf{z})$ th PWM of $W$ is formally defined by $\boldsymbol{\rho}_{M, \mathbf{z}}=\mathbf{E}\left(F_{\mathbf{\Psi}}(w)^{\mathbf{z}} W^{M}\right)$. Using equations (5) and (6), we can write

where

$$
f_{\underline{\Psi}}(w) F_{\underline{\Psi}}(w)^{\mathbf{z}}=\sum_{m=0}^{r} \sum_{\boldsymbol{\tau}=0}^{\infty} \sum_{k=0}^{2 \tau+1} \mathbf{c}_{m, \mathbf{\tau}, k}(k+1) b a^{b} t^{b-1} e^{-(a t)^{b}}\left[-e^{-(a t)^{b}}+1\right]^{k},
$$

$$
\begin{gathered}
\mathbf{c}_{m, \mathbf{\tau}, k}=\frac{2}{1+k} \alpha \lambda^{m}(1+\lambda)^{\mathbf{z}-m}(-1)^{m+j+k}\left(\begin{array}{c}
r \\
m
\end{array}\right)\left(\begin{array}{c}
1+2 j \\
k
\end{array}\right) \\
\times\left[\left(\begin{array}{c}
-1+(\mathbf{z}+m+1) \alpha \\
j
\end{array}\right)+\lambda\left(\begin{array}{c}
-1+(\mathbf{z}+m+1) \alpha \\
j
\end{array}\right)-2 \lambda\left(\begin{array}{c}
-1+(\mathbf{z}+m+2) \alpha \\
j
\end{array}\right)\right] .
\end{gathered}
$$

Then, using Theorem 1 the $(M, \mathbf{z})$ th PWM of $X$ can be expressed as

$$
\left.\boldsymbol{\rho}_{M, r}\right|_{[M>-b]}=\boldsymbol{\Gamma}\left(1+\frac{M}{b}\right) \sum_{h, m=0}^{\mathbf{z}} \sum_{\boldsymbol{\tau}=0}^{\infty} \sum_{k=0}^{2 \tau+1} \mathbf{C}_{\boldsymbol{\tau}, m, h, k}^{(M, 1+k)}
$$

where $\mathbf{C}_{\boldsymbol{\tau}, m, h, k}^{(M, 1+k)}=(1+k) \mathbf{c}_{m, \mathbf{\tau}, k} \frac{(-1)^{\mathfrak{\tau}}}{h ! a^{S}(\boldsymbol{\tau}+1)^{(M+b) / b}}\left(\begin{array}{l}k \\ h\end{array}\right)$.

\section{4 moments of residual life (MRL) and reversed residual life (MRRL)}

The $M^{\text {th }}$ MRL, say $\left.\mathbf{M R L}_{M}(\mathbf{z})\right|_{[M=1,2, \ldots \text { and } W>\mathbf{z}]}=\mathbf{E}\left[(W-\mathbf{z})^{M}\right]$, then

therefore

$$
\left.\mathbf{M R L}_{M}(\mathbf{z})\right|_{[M=1,2, \ldots \text { and } X>\mathbf{z}]}=\frac{\int_{\mathbf{z}}^{\infty} f_{\underline{\Psi}}(w)(w-\mathbf{z})^{M} d w}{1-F_{\underline{\Psi}}(\mathbf{z})},
$$

$$
\left.\mathbf{M R L}_{M}(\mathbf{z})\right|_{[M>-b]}=\frac{\Gamma\left(1+\frac{M}{b},(a \mathbf{z})^{b}\right)}{1-F(t)} \sum_{m, \mathbf{\tau}=0}^{\infty} \sum_{k=0}^{2 \tau} \mathbf{w}_{\mathbf{\tau}, k}^{*} a^{-M} \frac{k(-1)^{m}}{(m+1)^{(M+b) / b}}\left(\begin{array}{c}
-1+k \\
m
\end{array}\right),
$$

where $\mathbf{w}_{\tau, k}^{*}=\xi_{\tau, k} \sum_{r=0}^{M}\left(\begin{array}{c}M \\ r\end{array}\right)(-\mathbf{z})^{M-r}$. The $M^{t h}$ MRRL, say

then

$$
\left.\mathbf{M R R L}_{M}(\mathbf{z})\right|_{[\mathbf{z}>0, W \leq \mathbf{z} \text { and } M=1,2, \ldots]}=\mathbf{E}\left[(\mathbf{z}-W)^{M}\right],
$$

$\left.\operatorname{MRRL}_{M}(\mathbf{z})\right|_{[\mathbf{z}>0, X \leq \mathbf{z} \text { and } M=1,2, \ldots]}=\frac{1}{F_{\underline{\Psi}}(\mathbf{z})} \int_{0}^{\mathbf{z}}(\mathbf{z}-w)^{M} f_{\underline{\Psi}}(w) d w$. Then, the $n^{\text {th }}$ MRRL of $W$ becomes

$$
\left.\operatorname{MRRL}_{M}(\mathbf{z})\right|_{[M>-b]}=\frac{\gamma\left(1+\frac{M}{b},(a \mathbf{z})^{b}\right)}{F(\mathbf{z})} \sum_{m, \mathbf{\tau}=0}^{\infty} \sum_{k=0}^{2 \mathbf{\tau}} \mathbf{w}_{\mathbf{\tau}, k}^{* *} a^{-M} \frac{k(-1)^{m}}{(m+1)^{(M+b) / b}}\left(\begin{array}{c}
-1+k \\
m
\end{array}\right),
$$

where $\mathbf{w}_{\tau, k}^{* *}=\xi_{\mathbf{\tau}, k} \sum_{r=0}^{M}(-1)^{r}\left(\begin{array}{c}M \\ r\end{array}\right) \mathbf{z}^{M-r}$.

\subsection{Stress-strength model}

Let $W_{1}$ and $W_{2}$ be two independent rvs have TTL-W $\left(\lambda_{1}, \alpha, a, b\right)$ and TTL-W $\left(\lambda_{2}, \alpha, a, b\right)$ distributions. Then, the reliability is defined by $\mathbf{R}_{\left(W_{1}>W_{2}\right)}=\int_{0}^{\infty} F_{\lambda_{1}, \alpha, a, b}(w) f_{\lambda_{2}, \alpha, a, b}(w) d w$. Then, we can write

where

$$
\mathbf{R}_{\left(W_{1}>W_{2}\right)}=\sum_{\tau, w=0}^{\infty} \sum_{k=0}^{2 \tau} \sum_{m=0}^{2 w} \boldsymbol{\Omega}_{\boldsymbol{\tau}, w, k, m} .
$$

$$
\boldsymbol{\Omega}_{\boldsymbol{\tau}, w, k, m}=\frac{k}{k+m}(-1)^{\boldsymbol{\tau}+k+w+m}\left(\begin{array}{c}
2 h \\
k
\end{array}\right)\left[\left(\begin{array}{c}
\alpha \\
\boldsymbol{\tau}
\end{array}\right)+\lambda\left(\begin{array}{c}
\alpha \\
\boldsymbol{\tau}
\end{array}\right)-\lambda\left(\begin{array}{c}
2 \alpha \\
\boldsymbol{\tau}
\end{array}\right)\right]\left(\begin{array}{c}
2 w \\
m
\end{array}\right)\left[\left(\begin{array}{c}
\alpha \\
w
\end{array}\right)+\lambda\left(\begin{array}{c}
\alpha \\
w
\end{array}\right)-\lambda\left(\begin{array}{c}
2 \alpha \\
w
\end{array}\right)\right] .
$$




\subsection{Order statistics}

Let $W_{1}, \ldots, W_{n}$ be a random sample (R.S) of sixe $(n)$ from the TTL-W distribution and let $W_{(1)}, \ldots, W_{(n)}$ their corresponding order statistics. The P.D.F of $i-$ th order statistic, say $W_{i}:{ }_{n}$, can be written as

$$
f_{i}: n(w)=\sum_{h=0}^{n-i} \frac{(-1)^{h}\left(\begin{array}{c}
-i+n \\
j
\end{array}\right)}{B(i, n-i+1)} f_{\underline{\Psi}}(w) F_{\underline{\Psi}}(w)^{-1+h+i},
$$

where $B(.,$.$) is the beta function. Using (5) and (6) and (12), we get$

$$
f_{\underline{\Psi}}(w) F_{\underline{\Psi}}(w)^{-1+h+i}=\sum_{h=0}^{-1+h+i} \sum_{m=0}^{\infty} \sum_{k=0}^{2 m+1} \mathbf{v}_{h, m, k}(k+1) b a^{b} w^{b-1} e^{-(a t)^{b}}\left[-e^{-(a t w}+1\right]^{k},
$$

where

$$
\begin{gathered}
\mathbf{v}_{h, m, k}=\frac{2}{1+k} \alpha \lambda^{h}(1+\lambda)^{h+i-h-1}(-1)^{h+m+k}\left(\begin{array}{c}
-1+i+h \\
h
\end{array}\right) \\
\times\left(\begin{array}{c}
2 m+1 \\
k
\end{array}\right)\left\{\left(\begin{array}{c}
-1+(h+i+h) \alpha \\
m
\end{array}\right)+\lambda\left(\begin{array}{c}
-1+(h+i+h) \alpha \\
m
\end{array}\right)-2 \lambda\left(\begin{array}{c}
-1+(h+i+h+1) \alpha \\
m
\end{array}\right)\right\},
\end{gathered}
$$

the P.D.F of $W_{i: n}$ will be

$$
f_{i: n}(w)=\sum_{h=0}^{n-i} \frac{(-1)^{h}\left(\begin{array}{c}
n-i \\
j
\end{array}\right)}{B(i, n-i+1)} \sum_{\tau=0}^{h+i-1} \sum_{m=0}^{\infty} \sum_{k=0}^{2 m+1} \mathbf{v}_{\mathbf{\tau}, m, k}(k+1) b a^{b} w^{b-1} e^{-(a t w}\left[-e^{-(a w)^{b}}+1\right]^{k},
$$

the moments of $W_{i: n}$ will be

where

$$
\left.\mathbf{E}\left(W_{i: n}^{q}\right)\right|_{[r>-q]}=\Gamma\left(1+\frac{q}{b}\right) \sum_{w, m=0}^{\infty} \sum_{\tau=0}^{h+i-1} \sum_{k=0}^{2 m+1} \sum_{h=0}^{n-i} \mathbf{V}_{w, m, \tau, k, j}^{(q, 1+k)}
$$

\section{Estimation}

$$
\mathbf{V}_{w, m, \mathbf{\tau}, k, j}^{(q, 1+k)}=\mathbf{v}_{\mathbf{\tau}, m, k} \frac{(k+1)(-1)^{w+h} \Gamma(k+1)}{w ! a^{q} \mathrm{~B}(i, 1+n-i) \Gamma(1+k-w)}(w+1)^{-(q+b) / b}\left(\begin{array}{c}
-i+n \\
h
\end{array}\right) .
$$

\subsection{Maximum likelihood estimation}

Let $w_{1}, w_{2}, \ldots, w_{n}$ be a R.S from the TTL-W with parameters $\lambda, \alpha, a$ and $b$. Let $(\lambda, \alpha, a, b)^{T}=\underline{\Psi}$ be the $4 \times 1$ parameter vector. For getting the M-L-E of $\underline{\boldsymbol{\Psi}}$, we have the log-likelihood function $(\ell(\underline{\Psi}))$ as

$$
\begin{gathered}
\ell=\ell(\underline{\Psi})=n \log (2)+n \log \alpha+n \log b+n b \log a+(b-1) \sum_{i=1}^{n} \log \left(w_{i}\right) \\
-2 \sum_{i=1}^{n}\left(\alpha w_{i}\right)^{b}+(\alpha-1) \sum_{i=1}^{n} \log c_{i}+\sum_{i=1}^{n} \log z_{i},
\end{gathered}
$$

where $c_{i}=1-e^{-2\left(a w_{i}\right)^{b}}$ and $z_{i}=1+\lambda-2 \lambda c_{i}^{\alpha}$. The score vector components are

and

$$
\mathbf{U}_{\lambda}=\sum_{i=1}^{n} \frac{p_{i}}{z_{i}}, \mathbf{U}_{\alpha}=\frac{n}{\alpha}-\frac{2 b}{\alpha^{1-b}} \sum_{i=1}^{n} x_{i}^{b}+\sum_{i=1}^{n} \log c_{i}+\sum_{i=1}^{n} \frac{q_{i}}{z_{i}}, \mathbf{U}_{a}=\frac{n b}{a}+(\alpha-1) \sum_{i=1}^{n} \frac{w_{i}}{c_{i}}+\sum_{i=1}^{n} \frac{t_{i}}{z_{i}}
$$

where

$$
\mathbf{U}_{b}=\frac{n}{b}+n \log a+\sum_{i=1}^{n} \log \left(\mathbf{w}_{i}\right)-2 \sum_{i=1}^{n} \frac{\log \left(\alpha w_{i}\right)}{\left(\alpha x_{i}\right)^{-b}}+(\alpha-1) \sum_{i=1}^{n} \frac{m_{i}}{c_{i}}+\sum_{i=1}^{n} \frac{d_{i}}{z_{i}},
$$

$$
\begin{gathered}
\mathbf{w}_{i}=2 b a^{b-1} x_{i}^{b} e^{-2\left(a w_{i}\right)^{b}}, \quad m_{i}=2 e^{-2\left(a x_{i}\right)^{b}}\left(a x_{i}\right)^{b} \log \left(a w_{i}\right), \\
p_{i}=1-2 c_{i}^{\alpha}, q_{i}=-2 \lambda s_{i}^{\alpha} \log c_{i}, t_{i}=-2 \alpha \lambda w_{i} c_{i}^{\alpha-1} \text { and } d_{i}=-2 \alpha \lambda m_{i} c_{i}^{\alpha-1} .
\end{gathered}
$$

Generally, there are different forms of censorship such as type I, type II, etc. Here, we will consider a general case of multi-censorship in which there are $m$ subjects of which $m_{0}$ are known to have failed at the times $w_{1}, w_{2}, \ldots, w_{n_{0}-1}, w_{n_{0}}, n_{1}$ are known to have failed in the interval $\left[s_{j-1}, s_{j}\right], j=1,2, \ldots, m_{1}-1, m_{1}, m_{2}$ survived to a time $r_{j}, j=1,2, \ldots, m_{2}-1, m_{2}$ but not observed any longer. Where $m_{0}+m_{1}+m_{2}=m$ and that type I censoring and type II censoring are particular cases of multi-censoring. The $\log -\mathrm{L}\left(\ell_{m}(\underline{\boldsymbol{\Psi}})\right)$ for $\underline{\boldsymbol{\Psi}}$ is 


$$
\begin{aligned}
& \ell_{m}(\underline{\boldsymbol{\Psi}})=m_{0} \log 2+m_{0} \log \alpha+m_{0} \log b \\
& +m_{0} b \log a+(b-1) \sum_{i=1}^{m_{0}} \log \left(x_{i}\right)-2 \sum_{i=1}^{m_{0}}\left(\alpha w_{i}\right)^{b}+(\alpha-1) \sum_{i=1}^{m_{0}} \log \left[1-e^{-2\left(a w_{i}\right)^{b}}\right] \\
& +\sum_{i=1}^{m_{0}} \log \left(1-2 \lambda\left\{-e^{-2\left(a w_{i}\right)^{b}}+1\right\}^{\alpha}+\lambda\right) \\
& +\sum_{i=1}^{m_{1}} \log \left\{\begin{array}{c}
{\left[(1+\lambda)\left\{-e^{-2\left(a s_{i}\right)^{b}}+1\right\}^{\alpha}-\lambda\left\{-e^{-2\left(a s_{i}\right)^{b}}+1\right\}^{2 \alpha}\right]} \\
-\left[(1+\lambda)\left\{-e^{-2\left(a s_{i-1}\right)^{b}}+1\right\}^{\alpha}-\lambda\left\{-e^{-2\left(a s_{i-1}\right)^{b}}+1\right\}^{2 \alpha}\right]
\end{array}\right\} \\
& +\sum_{i=1}^{m_{2}} \log \left\{1-\left[(1+\lambda)\left\{-e^{-2\left(a r_{i}\right)^{b}}+1\right\}^{\alpha}-\lambda\left\{-e^{-2\left(a r_{i}\right)^{b}}+1\right\}^{2 \alpha}\right]\right\}
\end{aligned}
$$

For the normal equations see Appendix A.

\subsection{Least square (L-S) and weighted least square (W-L-S) estimation}

The theory of L-S estimation and W-L-S estimation is based on the minimization of the sum of the square of differences (SSD) of theoretical C.D.F and the empirical C.D.F. Suppose $F_{\underline{\Psi}}\left(w_{i: n}\right)$ denotes the distribution function of TTL-W distribution and if $w_{1}<w_{2}<\cdots<w_{n-1}<w_{n}$ be the $n$ ordered rs. The least square estimates are obtained by minimizing $\mathbf{L} \mathbf{s}(\underline{\boldsymbol{\Psi}})=\sum_{i=1}^{n}\left[F_{\underline{\Psi}}\left(w_{i}: n\right)-\vartheta_{i, 1+n}\right]^{2}$, where $\vartheta_{i, 1+n}=\frac{i}{1+n}$. Now using (5) we have

$$
\mathbf{L s}(\underline{\Psi})=\sum_{i=1}^{n}\left\{(1+\lambda)\left[-e^{-2\left(a w_{i}\right)^{b}}+1\right]^{\alpha}-\lambda\left[-e^{-2\left(a w_{i}\right)^{b}}+1\right]^{2 \alpha}-\vartheta_{i, 1+n}\right\}^{2} .
$$

The L-SE of the parameters are obtained by solving the following non-linear equations

$$
\begin{aligned}
& \sum_{i=1}^{n}\left\{(1+\lambda)\left[-e^{-2\left(a w_{i}\right)^{b}}+1\right]^{\alpha}-\lambda\left[-e^{-2\left(a w_{i}\right)^{b}}+1\right]^{2 \alpha}-\vartheta_{i, 1+n}\right\} C_{\lambda}\left(w_{i}, \underline{\Psi}\right)=0 \\
& \sum_{i=1}^{n}\left\{(1+\lambda)\left[-e^{-2\left(a w_{i}\right)^{b}}+1\right]^{\alpha}-\lambda\left[-e^{-2\left(a w_{i}\right)^{b}}+1\right]^{2 \alpha}-\vartheta_{i, 1+n}\right\} C_{\alpha}\left(w_{i}, \underline{\Psi}\right)=0 \\
& \sum_{i=1}^{n}\left\{(1+\lambda)\left[-e^{-2\left(a w_{i}\right)^{b}}+1\right]^{\alpha}-\lambda\left[-e^{-2\left(a w_{i}\right)^{b}}+1\right]^{2 \alpha}-\vartheta_{i, 1+n}\right\} C_{a}\left(w_{i}, \underline{\Psi}\right)=0
\end{aligned}
$$

and

$$
\sum_{i=1}^{n}\left\{(1+\lambda)\left[-e^{-2\left(a w_{i}\right)^{b}}+1\right]^{\alpha}-\lambda\left[-e^{-2\left(a w_{i}\right)^{b}}+1\right]^{2 \alpha}-\vartheta_{i, 1+n}\right\} C_{b}\left(w_{i}, \underline{\Psi}\right)=0,
$$

where $C_{\lambda}\left(w_{i}, \underline{\boldsymbol{\Psi}}\right), C_{\alpha}\left(w_{i}, \underline{\boldsymbol{\Psi}}\right), C_{a}\left(w_{i}, \underline{\boldsymbol{\Psi}}\right)$ and $C_{b}\left(w_{i}, \underline{\boldsymbol{\Psi}}\right)$ are the values of the first derivatives of the C.D.F w.r.t. parameters of TTL-W distribution. The L-S estimates of the parameters $\lambda, \alpha, a, b$ are obtained solving the above simultaneous equations by using the numerical approximation techniques. The W-L-S estimations (W-L-SEs) are obtained by minimizing the given form of equation with respect to the parameters

$$
\mathbf{W L S}(\underline{\Psi})=\sum_{i=1}^{n} \boldsymbol{w}_{(i)}\left[F_{\underline{\Psi}}\left(w_{i}: n\right)-\vartheta_{i, 1+n}\right]^{2}
$$

The W-L-SE of the parameters are obtained by solving the following non-linear equations;

$$
\sum_{i=1}^{n}\left\{(1+\lambda)\left[-e^{-2\left(a w_{i}\right)^{b}}+1\right]^{\alpha}-\lambda\left[-e^{-2\left(a w_{i}\right)^{b}}+1\right]^{2 \alpha}-\vartheta_{i, 1+n}\right\} \boldsymbol{w}_{(i)} C_{\lambda}\left(w_{i}, \underline{\Psi}\right)=0,
$$




$$
\begin{aligned}
& \sum_{i=1}^{n}\left\{(1+\lambda)\left[-e^{-2\left(a w_{i}\right)^{b}}+1\right]^{\alpha}-\lambda\left[-e^{-2\left(a w_{i}\right)^{b}}+1\right]^{2 \alpha}-\vartheta_{i, 1+n}\right\} \boldsymbol{w}_{(i)} C_{\alpha}\left(w_{i}, \underline{\Psi}\right)=0 \\
& \sum_{i=1}^{n}\left\{(1+\lambda)\left[-e^{-2\left(a w_{i}\right)^{b}}+1\right]^{\alpha}-\lambda\left[-e^{-2\left(a w_{i}\right)^{b}}+1\right]^{2 \alpha}-\vartheta_{i, 1+n}\right\} \boldsymbol{w}_{(i)} C_{a}\left(w_{i}, \underline{\Psi}\right)=0
\end{aligned}
$$

and

$$
\sum_{i=1}^{n}\left\{(1+\lambda)\left[-e^{-2\left(a w_{i}\right)^{b}}+1\right]^{\alpha}-\lambda\left[-e^{-2\left(a w_{i}\right)^{b}}+1\right]^{2 \alpha}-\vartheta_{i, 1+n}\right\} \boldsymbol{w}_{(i)} C_{b}\left(w_{i}, \underline{\Psi}\right)=0,
$$

where $C_{\lambda}\left(w_{i}, \underline{\boldsymbol{\Psi}}\right), C_{\alpha}\left(w_{i}, \underline{\boldsymbol{\Psi}}\right), C_{a}\left(w_{i}, \underline{\boldsymbol{\Psi}}\right)$ and $C_{b}\left(w_{i}, \underline{\boldsymbol{\Psi}}\right)$ are the values of first derivatives of the C.D.F of TTL-W distribution and $\boldsymbol{w}_{(i)}=\left[(n+1)^{2}(n+2)\right] \div[i(n-i+1)]$.

\subsection{Method of Cramer-Von-Mises}

The Crammer-Von Mises estimates of the parameters (see MacDonald (1971)) are obtained by minimizing the following expression w.r.t. to the parameters as

where $V_{2 i-1,2 n}=\frac{2 i-1}{2 n}$, and

$$
\operatorname{CVM}(\lambda, \alpha, a, b)=\frac{1}{12 n}+\sum_{i=1}^{n}\left[-V_{2 i-1,2 n}+F_{\lambda, \alpha, a, b}\left(w_{i}: n\right)\right]^{2},
$$

$$
\operatorname{CVM}(\lambda, \alpha, a, b)=\sum_{i=1}^{n}\left\{(1+\lambda)\left[-e^{-2\left(a w_{i}\right)^{b}}+1\right]^{\alpha}-\lambda\left[-e^{-2\left(a w_{i}\right)^{b}}+1\right]^{2 \alpha}-V_{2 i-1,2 n}\right\}^{2} .
$$

The Cramer-Von-Mises estimators (C-V-ME) of the parameters are obtained by solving the following non-linear equations

$$
\begin{aligned}
& \sum_{i=1}^{n}\left\{(1+\lambda)\left[-e^{-2\left(a w_{i}\right)^{b}}+1\right]^{\alpha}-\lambda\left[-e^{-2\left(a w_{i}\right)^{b}}+1\right]^{2 \alpha}-V_{2 i-1,2 n}\right\} C_{\lambda}\left(w_{i}, \underline{\Psi}\right)=0, \\
& \sum_{i=1}^{n}\left\{(1+\lambda)\left[-e^{-2\left(a w_{i}\right)^{b}}+1\right]^{\alpha}-\lambda\left[-e^{-2\left(a w_{i}\right)^{b}}+1\right]^{2 \alpha}-V_{2 i-1,2 n}\right\} C_{\alpha}\left(w_{i}, \underline{\boldsymbol{\Psi}}\right)=0, \\
& \sum_{i=1}^{n}\left\{(1+\lambda)\left[-e^{-2\left(a w_{i}\right)^{b}}+1\right]^{\alpha}-\lambda\left[-e^{-2\left(a w_{i}\right)^{b}}+1\right]^{2 \alpha}-V_{2 i-1,2 n}\right\} C_{a}\left(w_{i}, \underline{\Psi}\right)=0,
\end{aligned}
$$

and

$$
\sum_{i=1}^{n}\left\{(1+\lambda)\left[-e^{-2\left(a w_{i}\right)^{b}}+1\right]^{\alpha}-\lambda\left[-e^{-2\left(a w_{i}\right)^{b}}+1\right]^{2 \alpha}-V_{2 i-1,2 n}\right\} C_{b}\left(w_{i}, \underline{\Psi}\right)=0,
$$

where $C_{\lambda}\left(w_{i}, \underline{\boldsymbol{\Psi}}\right), C_{\alpha}\left(w_{i}, \underline{\boldsymbol{\Psi}}\right), C_{a}\left(w_{i}, \underline{\boldsymbol{\Psi}}\right)$ and $C_{b}\left(w_{i}, \underline{\boldsymbol{\Psi}}\right)$ are the values of the first derivatives of the C.D.F of TTL-W distribution with respect to $\lambda, \alpha, a, b$ respectively.

\section{Real data analysis}

\subsection{Application 1}

The 1st data set consists of 100 observations of carbon fibers breaking stress given by Nicholas and Padgett (2006). The data are $(0.39,0.850,1.08,2.590,2.67,2.730,2.74,1.250,1.47,1.570,1.61,1.610,1.69,1.80,1.840,1.87,1.890$, $2.03,2.03,2.05,2.120,2.35,2.41,2.430,2.480,2.50,2.53,2.55,2.550,2.56,2.790,2.870,2.88,2.930,2.950,2.96$, 2.97, 3.090, 2.81, 2.82, 2.850, 3.11, 3.110, 3.15, 3.150, 3.270, 3.28, 3.31, 3.31, 3.33, 3.39, 3.190, 3.22, 3.22, 3.39, $3.56,3.60,3.65,3.68,3.70,3.75,4.20,4.38,4.420,4.70,4.90)$. Table 3 gives the values of estimators of $\alpha, \lambda, a$ and $b$, the KS test statistics and its p-value for new for the four different estimation methods. 
Table 3. The values of estimators, KS, p-values for the first application.

\begin{tabular}{l|llllll}
\hline Method & $\alpha$ & $\lambda$ & $\mathrm{a}$ & $\mathrm{b}$ & $\mathrm{KS}$ & $\mathrm{p}$-value \\
\hline ML & 0.75959 & 0.17308 & 0.25205 & 4.18957 & 0.08173 & 0.77000 \\
L-S & 0.43393 & 0.57147 & 0.24279 & 7.69659 & 0.05988 & 0.97195 \\
W-L-S & 0.54471 & 0.36649 & 0.24784 & 6.01142 & 0.06591 & 0.93667 \\
C-V-M & $\mathbf{0 . 4 3 9 0 3}$ & $\mathbf{0 . 5 9 0 7 5}$ & $\mathbf{0 . 2 4 4 2 1}$ & $\mathbf{7 . 8 2 0 1 4}$ & $\mathbf{0 . 0 5 8 7 6}$ & $\mathbf{0 . 9 7 6 6 2}$ \\
Bootstrap & 0.58086 & 0.14799 & 0.23655 & 5.92953 & 0.19592 & 0.01260 \\
\hline
\end{tabular}

From Table 3, we conclude that the C-V-M method is the best method for modelling the carbon fibers with $\mathrm{KS}=0.05876$ and biggest $\mathrm{p}$-value $=0.97662$. However, all other methods performed well.

\subsection{Application 2}

Data 2 nd data set $(65,56,26,22,1,1,5,65,16,22,3,4,2,3,56,65,17,7,156,8,4,3,30,4,100,134,16,108,121$, $4,39,143,43)$ called leukemia data. This real data set gives the survival times, in weeks, of 33 patients suffering from acute Myelogenous Leukemia (see Feigl and Zelen (1965)).

Table 4. The values of estimators, KS, p-values for the second application.

\begin{tabular}{l|llllll}
\hline Method & $\alpha$ & $\lambda$ & $\mathrm{a}$ & $\mathrm{b}$ & $\mathrm{KS}$ & $\mathrm{p}$-value \\
\hline ML & $\mathbf{1 6 2 . 7 3 1 8 8}$ & $\mathbf{0 . 3 7 0 4 8}$ & $\mathbf{2 9 2 . 2 4 0 5 4}$ & $\mathbf{0 . 1 0 6 8 2}$ & $\mathbf{0 . 1 8 9 1 9}$ & $\mathbf{0 . 5 7 6 9 0}$ \\
L-S & 141.25302 & 0.26132 & 121.83737 & 0.11267 & 0.22642 & 0.34809 \\
W-L-S & 140.45773 & 0.27289 & 133.10529 & 0.11413 & 0.19373 & 0.54616 \\
C-V-M & 143.59549 & 0.21879 & 127.52666 & 0.11336 & 0.22312 & 0.36580 \\
Bootstrap & 123.95395 & 0.49129 & 155.62680 & 0.11419 & 0.28442 & 0.12777 \\
\hline
\end{tabular}

From Table 4, we conclude that the ML method is the best method for modeling the Leukemia data with KS=0.18919 and biggest $\mathrm{p}$-value $=0.57690$. However, all other methods performed well. For comparing with existing competitive models, many real data sets can be found in Refaie (2018), Elbiely and Yousof (2018, 2019), Goual and Yousof (2019), Al-Babtain et al. (2020a,b), Goual et al. (2019, 2020), Hamedani et al. (2019), Ibrahim (2019), Ibrahim et al. (2019, 2020), Korkmaz et al. (2018), Korkmaz et al. (2017, 2019a,b), Mansour et al. (2020a,b), Yadav et al. (2020) and $\operatorname{Ibrahim}(2020 \mathrm{a}, \mathrm{b})$.

\section{Monte Carlo (M-C) Simulations}

A M-C simulation study is performed in this Section to compare the performance of the different estimators of the unknown parameters of the new distribution via their mean squared errors (MSEs). All the computations in this section are done by Mathcad program Version 15.0. Samples of size 1000 is generated from the new distribution where $n$ $=20,50,100$ and 200. The average values (AVs) and MSEs of M-L-Es, L-SEs, W-L-SEs, C-V-Ms and Bootstrapping are obtained and reported in in Tables 5-8. From Tables 5-8, all the estimates have the property of consistency, i.e., the MSEs decrease as sample size $n$ increase.

Table 5: AVs and MSEs for $n=20$.

\begin{tabular}{c|ccccc}
\hline Parameters & M-L-E & L-S & W-L-S & C-V-M & Bootstrap \\
\hline$\alpha=2.3$ & $2.4(0.2640)$ & $2.3(0.328)$ & $2.3(0.31)$ & $2.4(0.35)$ & $2.34(0.241)$ \\
$\lambda=0.5$ & $0.5(0.371)$ & $0.6(0.210)$ & $0.56(0.21)$ & $0.50(0.16)$ & $0.7(0.83)$ \\
$\mathrm{a}=0.5$ & $0.51(0.0384)$ & $0.56(0.091)$ & $0.58(0.152)$ & $0.57(0.155)$ & $0.59(0.071)$ \\
$\mathrm{b}=0.5$ & $0.51(0.00648)$ & $0.6(0.128)$ & $0.55(0.081)$ & $0.6(0.09)$ & $0.51(0.007)$ \\
\hline$\alpha=1.2$ & $1.2(0.0477)$ & $1.2(0.060)$ & $1.2(0.0576)$ & $1.2(0.057)$ & $1.2(0.191)$ \\
$\lambda=0.9$ & $0.84(0.1127)$ & $0.96(0.214)$ & $0.95(0.197)$ & $0.92(0.190)$ & $0.95(0.193)$ \\
$\mathrm{a}=1.6$ & $1.7(0.2864)$ & $1.7(0.421)$ & $1.74(0.429)$ & $1.7(0.30)$ & $1.9(0.50)$ \\
$\mathrm{b}=0.8$ & $0.81(0.0138)$ & $0.82(0.039)$ & $0.81(0.022)$ & $0.82(0.025)$ & $0.79(0.014)$ \\
\hline$\alpha=1.9$ & $1.54(0.1563)$ & $1.9(0.055)$ & $1.92(0.045)$ & $1.9(0.048)$ & $1.18(0.190)$ \\
$\lambda=2.0$ & $1.95(0.17)$ & $2.02(0.195)$ & $2(0.16)$ & $2.01(0.181)$ & $2.05(0.19)$ \\
$\mathrm{a}=0.8$ & $0.92(0.0172)$ & $0.80(0.005)$ & $0.80(0.003)$ & $0.80(0.004)$ & $1.87(0.50)$ \\
$\mathrm{b}=1.6$ & $0.1 .32(0.0872)$ & $1.6(0.035)$ & $1.62(0.032)$ & $1.60(0.030)$ & $0.79(0.014)$ \\
\hline
\end{tabular}


Table 6: AVs and MSEs for $n=50$.

\begin{tabular}{c|ccccc}
\hline Parameters & M-L-E & L-S & W-L-S & C-V-M & Bootstrap \\
\hline$\alpha=2.3$ & $2.3(0.10599)$ & $2.32(0.12)$ & $2.3(0.092)$ & $2.33(0.117)$ & $2.03(0.140)$ \\
$\lambda=0.5$ & $0.44(0.08372)$ & $0.52(0.079)$ & $0.51(0.072)$ & $0.51(0.078)$ & $0.77(0.180)$ \\
$\mathrm{a}=0.5$ & $0.50(0.1553)$ & $0.52(0.0181)$ & $0.52(0.016)$ & $0.51(0.017)$ & $0.68(0.066)$ \\
$\mathrm{b}=0.5$ & $0.504(0.00284)$ & $0.51(0.011)$ & $0.52(0.018)$ & $0.51(0.011)$ & $0.46(0.004)$ \\
\hline$\alpha=1.2$ & $1.21(0.01771)$ & $1.21(0.023)$ & $1.2(0.019)$ & $1.2(0.023)$ & $1.07(0.032)$ \\
$\lambda=0.9$ & $0.85(0.1098)$ & $0.92(0.082)$ & $0.91(0.069)$ & $0.92(0.163)$ & $1.29(0.503)$ \\
$\mathrm{a}=1.6$ & $1.60(0.1083)$ & $1.6(0.124)$ & $1.6(0.108)$ & $1.64(0.121)$ & $2.08(0.452)$ \\
$\mathrm{b}=0.8$ & $0.81(0.0052)$ & $0.81(0.0080)$ & $0.81(0.0066)$ & $0.81(0.008)$ & $0.73(0.010)$ \\
\hline$\alpha=1.9$ & $1.5(0.1362)$ & $1.9(0.022)$ & $1.91(0.015)$ & $1.9(0.022)$ & $1.44(0.224)$ \\
$\lambda=2.0$ & $1.985(0.086)$ & $2(0.075)$ & $1.99(0.032)$ & $2.01(0.089)$ & $1.86(0.14)$ \\
$\mathrm{a}=0.8$ & $0.91(0.0135)$ & $0.80(0.0017)$ & $0.80(0.00098)$ & $0.80(0.002)$ & $0.95(0.024)$ \\
$\mathrm{b}=1.6$ & $1.33(0.0767)$ & $1.6(0.014)$ & $1.61(0.010)$ & $1.6(0.013)$ & $1.25(0.130)$ \\
\hline
\end{tabular}

Table 7: AVs and MSEs for $n=100$.

\begin{tabular}{c|ccccc}
\hline Parameters & M-L-E & L-S & W-L-S & C-V-M & Bootstrap \\
\hline$\alpha=2.3$ & $2.3(0.0458)$ & $2.3(0.0519)$ & $2.3(0.0461)$ & $2.31(0.052)$ & $2.01(0.084)$ \\
$\lambda=0.5$ & $0.45(0.038)$ & $0.50(0.035)$ & $0.502(0.035)$ & $0.51(0.04)$ & $0.69(0.081)$ \\
$\mathrm{a}=0.5$ & $0.50(0.007)$ & $0.51(0.0068)$ & $0.51(0.006)$ & $0.51(0.007)$ & $0.62(0.030)$ \\
$\mathrm{b}=0.5$ & $0.502(0.001)$ & $0.51(0.0036)$ & $0.51(0.002)$ & $0.51(0.004)$ & $0.44(0.005)$ \\
\hline$\alpha=1.2$ & $1.21(0.0083)$ & $1.2(0.011)$ & $1.2(0.0095)$ & $1.2(0.01)$ & $0.62(0.030)$ \\
$\lambda=0.9$ & $0.85(0.0441)$ & $0.90(0.038)$ & $0.90(0.034)$ & $0.91(0.04)$ & $0.91(0.060)$ \\
$\mathrm{a}=1.6$ & $1.59(0.0513)$ & $1.62(0.051)$ & $1.6(0.0490)$ & $1.6(0.050)$ & $1.7(0.0740)$ \\
$\mathrm{b}=0.8$ & $0.80(0.0025)$ & $0.80(0.0036)$ & $0.50(0.001)$ & $0.8(0.003)$ & $0.79(0.003)$ \\
\hline$\alpha=1.9$ & $1.54(0.1340)$ & $1.91(0.0104)$ & $1.9(0.0060)$ & $1.9(.0 .010)$ & $1.52(0.150)$ \\
$\lambda=2.0$ & $1.92(0.0540)$ & $1.99(0.036)$ & $1.99(0.012)$ & $2.01(0.040)$ & $1.94(0.064)$ \\
$\mathrm{a}=0.8$ & $0.91(0.0128)$ & $0.79(.0 .0008)$ & $0.80(0.642)$ & $0.8(.0 .008)$ & $0.92(0.0140)$ \\
$\mathrm{b}=1.6$ & $1.33(0.0753)$ & $1.61(0.0065)$ & $1.61(0.0046)$ & $1.6(0.006)$ & $1.31(0.0841)$ \\
\hline
\end{tabular}

Table 8: AVs and MSEs for $n=200$.

\begin{tabular}{c|ccccc}
\hline Parameters & M-L-E & L-S & W-L-S & C-V-M & Bootstrap \\
\hline$\alpha=2.3$ & $2.3(0.02526)$ & $2.31(0.0249)$ & $2.3(0.022)$ & $2.3(0.0252)$ & $2.06(0.074)$ \\
$\lambda=0.5$ & $0.49(0.0212)$ & $0.502(0.017)$ & $0.50(0.017)$ & $0.51(0.018)$ & $0.61(0.026)$ \\
$\mathrm{a}=0.5$ & $0.50(0.0044)$ & $0.503(0.033)$ & $0.50(0.003)$ & $0.50(0.034)$ & $0.68(0.041)$ \\
$\mathrm{b}=0.5$ & $0.50(0.0006)$ & $0.503(0.001)$ & $0.50(0.001)$ & $0.51(0.001)$ & $0.46(0.0018)$ \\
\hline$\alpha=1.2$ & $1.2(0.0047)$ & $1.2(0.0051)$ & $1.2(0.0046)$ & $1.2(0.0051)$ & $1.06(0.021)$ \\
$\lambda=0.9$ & $0.87(0.022)$ & $0.90(0.0187)$ & $0.9(0.017)$ & $0.91(0.019)$ & $1.07(0.063)$ \\
$\mathrm{a}=1.6$ & $1.6(0.0298)$ & $1.6(0.0249)$ & $1.6(0.0232)$ & $1.6(0.031)$ & $2.07(0.27)$ \\
$\mathrm{b}=0.8$ & $0.80(0.0014)$ & $0.80(0.0017)$ & $0.80(0.001)$ & $0.80(0.002)$ & $0.73(0.0064)$ \\
\hline$\alpha=1.9$ & $1.54(0.1328)$ & $1.90(0.0051)$ & $1.9(0.0029)$ & $1.90(0.005)$ & $1.4(0.242)$ \\
$\lambda=2.0$ & $2.13(0.0164)$ & $1.99(0.0178)$ & $1.99(0.005)$ & $2(0.0180)$ & $0.96(0.0271)$ \\
$\mathrm{a}=0.8$ & $0.91(0.013)$ & $0.80(0.0003)$ & $0.80(0.000)$ & $0.80(0.0004)$ & $0.96(0.027)$ \\
$\mathrm{b}=1.6$ & $1.33(0.075)$ & $1.6(0.0031)$ & $1.6(0.0021)$ & $1.6(0.0030)$ & $1.2(0.14)$ \\
\hline
\end{tabular}

\section{Conclusions}

In this paper we focus on proposing a new lifetime Weibull model called the transmuted Topp-Leone Weibull model and studying its mathematical properties. The skewness of the proposed distribution can range in the interval $(-0.68$, 5.1) and this means that the new model can be left and right skewed, whereas the kurtosis of the proposed distribution varies in the interval $(-398.1,54.1)$. The estimation of its unknown parameters is carried out by considering different method of estimation. The performances of all methods are studied by two real data sets and a Monte Carlo simulation. 
The Cramer-Von Mises method is the best method for modeling the carbon fibers data with $\mathrm{KS}=0.05876$ and biggest p-value $=0.97662$. The maximum likelihood method is the best method for modeling the Leukemia data with $\mathrm{KS}=0.18919$ and biggest $\mathrm{p}$-value $=0.57690$, however all other methods performed well.

\section{References}

1. Al-Babtain, A. A., Elbatal, I. and Yousof, H. M. (2020a). A new three parameter Fréchet model with mathematical properties and applications. Journal of Taibah University for Science, 14(1), 265-278.

2. Al-babtain, A. A., Elbatal, I. and Yousof, H. M. (2020b). A New Flexible Three-Parameter Model: Properties, Clayton Copula, and Modeling Real Data. Symmetry, 12(3), 440.

3. Alizadeh, M., Ghosh, I., Yousof, H. M., Rasekhi, M. and Hamedani G. G. (2017). The generalized odd generalized exponential family of distributions: properties, characterizations and applications, Journal of Data Science $15,443-466$.

4. Almamy, J. A., Ibrahim, M., Eliwa, M. S., Al-mualim, S. and Yousof, H. M. (2018). The two-parameter odd Lindley Weibull lifetime model with properties and applications. International Journal of Statistics and Probability, 7(4), 1927-7040.

5. Aryal, G. R., Ortega, E. M., Hamedani, G. G. and Yousof, H. M. (2017). The Topp Leone Generated Weibull distribution: regression model, characterizations and applications. International Journal of Statistics and Probability, 6, 126-141.

6. Aryal, G. R. and Yousof, H. M. (2017). The exponentiated generalized-G Poisson family of distributions. Economic Quality Control, 32(1), 1-17.

7. Brito, E., Cordeiro, G. M., Yousof, H. M., Alizadeh, M. and Silva, G. O. (2017). Topp-Leone Odd LogLogistic Family of Distributions. Journal of Statistical Computation and Simulation, 87(15), 3040-3058.

8. Cordeiro, G. M., Yousof, H. M., Ramires, T. G. and Ortega, E. M. M. (2018). The Burr XII system of densities: properties, regression model and applications. Journal of Statistical Computation and Simulation, 88(3), 432-456.

9. Elbiely, M. M. and Yousof, H. M. (2018). A New Extension of the Lomax Distribution and its Applications, Journal of Statistics and Applications, 2(1), 18-34.

10. Elbiely, M. M. and Yousof, H. M. (2019). A New Inverse Weibull Distribution: Properties and Applications. Journal of Mathematics and Statistics, 15(1), 30-43.

11. Farlie, D. J. G. (1960) The performance of some correlation coefficients for a general bivariate distribution. Biometrika, 47, 307-323.

12. Feigl, P., and Zelen, M. (1965). Estimation of Exponential Survival Probabilities with Concomitant Information, Biometrics, 21, 826-838, www.jstor.org/stable/2528247.

13. Goual, H., Yousof, H. M. and Ali, M. M. (2019). Validation of the odd Lindley exponentiated exponential by a modified goodness of fit test with applications to censored and complete data. Pakistan Journal of Statistics and Operation Research, 745-771.

14. Goual, H. and Yousof, H. M. (2019). Validation of Burr XII inverse Rayleigh model via a modified chisquared goodness-of-fit test. Journal of Applied Statistics, 47(1), 1-32.

15. Goual, H., Yousof, H. M. and Ali, M. M. (2020). Lomax inverse Weibull model: properties, applications and a modified Chi-squared goodness-of-fit test for validation, Journal of Nonlinear Science and Applications. 13(6), 330353.

16. Gumbel, E. J. (1961). Bivariate logistic distributions. Journal of the American Statistical Association, 56(294), 335-349.

17. Gumbel, E. J. (1960) Bivariate exponential distributions. Journ. Amer. Statist. Assoc., 55, 698-707.

18. Hamedani, G. G., Rasekhi, M., Najibi, S., Yousof, H. M. and Alizadeh, M. (2019). Type II general exponential class of distributions. Pakistan Journal of Statistics and Operation Research, 503-523.

19. Hamedani, G. G., Yousof, H. M., Rasekhi, M., Alizadeh, M. and Najibi, S. M. (2018). Type I general exponential class of distributions. Pakistan Journal of Statistics and Operation Research, 39-55.

20. Ibrahim, M. (2019). A new extended Fréchet distribution: properties and estimation. Pak. J. Stat. Oper. Res.,15 (3), 773-796.

21. Ibrahim, M. (2020a). The compound Poisson Rayleigh Burr XII distribution: properties and applications. Journal of Applied Probability and Statistics, 15(1), 73-97. 
22. Ibrahim, M. (2020b). The generalized odd Log-logistic Nadarajah Haghighi distribution: statistical properties and different methods of estimation. Journal of Applied Probability and Statistics, 15(2), 61-84.

23. Ibrahim, M., Altun, E. and Yousof, H. M. (2020). A new distribution for modeling lifetime data with different methods of estimation and censored regression modeling. Statistics, Optimization \& Information Computing, 8(2), 610-630.

24. Ibrahim, M., Yadav, A. S. Yousof, H. M., Goual, H. and Hamedani, G. G. (2019). A new extension of Lindley distribution: modified validation test, characterizations and different methods of estimation, Communications for Statistical Applications and Methods, 26(5), 473-495.

25. Johnson, N. L. and Kotz, S. (1975) On some generalized Farlie- Gumbel- Morgenstern distributions. Commun. Stat. Theory, 4, 415-427.

26. Johnson, N. L. and Kotz, S. (1977) On some generalized Farlie-Gumbel-Morgenstern distributions- II: Regression, correlation and further generalizations. Commun. Stat.Theory, 6, 485-496.

27. Korkmaz, M. Ç., Alizadeh, M., Yousof, H. M., \& Butt, N. S. (2018). The generalized odd Weibull generated family of distributions: statistical properties and applications. Pakistan Journal of Statistics and Operation Research, 541-556.

28. Korkmaz, M. C., Altun, E., Alizadeh, M. and Yousof, H. M. (2019a). A new flexible lifetime model with log-location regression modeling, properties and applications. Journal of Statistics \& Management Systems, 22(5), 870-891.

29. Korkmaz, M. C., Altun, E., Yousof, H. M. and Hamedani G. G. (2019b). The Odd Power Lindley Generator of Probability Distributions: Properties, Characterizations and Regression Modeling. International Journal of Statistics and Probability, 8(2). 70-89.

30. Korkmaz, M. C. Yousof, H. M. and Ali, M. M. (2017). Some theoretical and computational aspects of the odd Lindley Fréchet distribution, Journal of Statisticians: Statistics and Actuarial Sciences, 2, 129-140.

31. Mansour, M., Rasekhi, M., Ibrahim, M., Aidi, K., Yousof, H. M. and Elrazik, E. A. (2020a). A New Parametric Life Distribution with Modified Bagdonavičius-Nikulin Goodness-of-Fit Test for Censored Validation, Properties, Applications, and Different Estimation Methods. Entropy, 22(5), 592.

32. Mansour, M., Yousof, H. M., Shehata, W. A. M. and Ibrahim, M. (2020b). A new two parameter Burr XII distribution: properties,copula, different estimation methods and modeling acute bone cancer data, Journal of Nonlinear Science and Applications, 13, 223-238.

33. Merovci, F., Alizadeh, M., Yousof, H. M. and Hamedani G. G. (2017). The exponentiated transmuted-G family of distributions: theory and applications. Communications in Statistics-Theory and Methods, 46(21), 1080010822.

34. NichoL-S, M. D, Padgett, W. J. (2006). A Bootstrap control chart for Weibull percentiles. Quality and Reliability Engineering International, 22, 141-151.

35. Pougaza, D. B. and Djafari, M. A. (2011). Maximum entropies copulas. Proceedings of the 30th international workshop on Bayesian inference and maximum Entropy methods in Science and Engineering, 329-336.

36. Refaie, M. K. (2018). Extended Poisson-Exponentiated Weibull distribution: theoretical and computational aspects. Pak. J. Statist, 34(6), 513-530.

37. Rezaei, S., Sadr, B. B., Alizadeh, M. and Nadarajah, S. (2017). Topp-Leone generated family of distributions: Properties and applications. Communications in Statistics-Theory and Methods, 46(6), 2893-2909.

38. Rodriguez-Lallena, J. A. and Ubeda-Flores, M. (2004). A new class of bivariate copulas. Statistics and Probability Letters, 66, 315-25.

39. Shaw, W.T., Buckley, I.R.C. (2007). The alchemy of probability distributions: beyond Gram-Charlier expansions and a skew-kurtotic-normal distribution from a rank transmutation map. arXiv preprint arXiv:0901.0434.

40. Yadav, A. S., Goual, H., Alotaibi, R. M., Ali, M. M. and Yousof, H. M. (2020). Validation of the ToppLeone-Lomax model via a modified Nikulin-Rao-Robson goodness-of-fit test with different methods of estimation. Symmetry, 12(1), 57.

41. Yousof, H. M., Afify, A. Z., Alizadeh, M., Butt, N. S., Hamedani, G. G. and Ali, M. M. (2015). The transmuted exponentiated generalized-G family of distributions. Pakistan Journal of Statistics and Operation Research, 11 (4), 441-464.

42. Yousof, H. M., Afify, A. Z., Cordeiro, G. M., Alzaatreh, A., and Ahsanullah, M. (2017a). A new fourparameter Weibull model for lifetime data. Journal of Statistical Theory and Applications, 16(4), $448-466$. 
43. Yousof, H. M., Alizadeh, M., Jahanshahiand, S. M. A., Ramires, T. G., Ghosh, I. and Hamedani G. G. (2017b). The transmuted Topp-Leone $\mathrm{G}$ family of distributions: theory, characterizations and applications. Journal of Data Science. 15, 723-740.

44. Yousof, H. M., Altun, E., Ramires, T. G., Alizadeh, M. and Rasekhi, M. (2018). A new family of distributions with properties, regression models and applications. Journal of Statistics and Management Systems, 21(1), 163-188.

\section{Appendix A:}

$$
\begin{aligned}
& \frac{\partial}{\partial \lambda} \ell_{m}(\underline{\Psi})=\sum_{i=1}^{m_{0}} \frac{1-2\left\{1-e^{-2\left(a w_{i}\right)^{b}}\right\}^{\alpha}}{1-2 \lambda\left\{1-e^{-2\left(a w_{i}\right)^{b}}\right\}^{\alpha}+\lambda}+\sum_{i=1}^{m_{1}} \frac{\left[\mathbf{A}^{(\lambda)}\left(s_{i}\right)-\mathbf{A}^{(\lambda)}\left(s_{i-1}\right)\right]}{\left\{\left[\begin{array}{c}
(1+\lambda)\left\{1-e^{-2\left(a s_{i}\right)^{b}}\right\}^{\alpha} \\
-\lambda\left\{1-e^{-2\left(a s_{i}\right)^{b}}\right\}^{2 \alpha}
\end{array}\right]-\left[\begin{array}{c}
(1+\lambda)\left\{1-e^{-2\left(a s_{i-1}\right)^{b}}\right\}^{\alpha} \\
-\lambda\left\{1-e^{-2\left(a s_{i-1}\right)^{b}}\right\}^{2 \alpha}
\end{array}\right]\right\}} \\
& +\sum_{i=1}^{m_{2}} \frac{\mathbf{A}^{(\lambda)}\left(r_{i}\right)}{1-\left[(1+\lambda)\left\{1-e^{-2\left(a r_{i}\right)^{b}}\right\}^{\alpha}\right]-\lambda\left\{1-e^{-2\left(a r_{i}\right)^{b}}\right\}^{2 \alpha}} \\
& \frac{\partial \ell_{m}(\underline{\Psi})}{\partial \alpha}=\frac{m_{0}}{\alpha}-\frac{2 b}{\alpha^{1-b}} \sum_{i=1}^{m_{0}} x_{i}^{b}+\sum_{i=1}^{m_{0}} \log \left\{1-e^{-2\left(a x_{i}\right)^{b}}\right\}+\sum_{i=1}^{m_{0}} \frac{-2 \lambda\left\{1-e^{-2\left(a w_{i}\right)^{b}}\right\}^{\alpha} \log \left\{1-e^{-2\left(a w_{i}\right)^{b}}\right\}}{1+\lambda-2 \lambda\left\{1-e^{-2\left(a w_{i}\right)^{b}}\right\}^{\alpha}} \\
& +\sum_{i=1}^{m_{1}} \frac{\left[\mathbf{B}^{(\alpha)}\left(s_{i}\right)-\mathbf{B}^{(\alpha)}\left(s_{i-1}\right)\right]}{\left\{\left[\begin{array}{c}
(1+\lambda)\left\{1-e^{-2\left(a s_{i}\right)^{b}}\right\}^{\alpha} \\
-\lambda\left\{1-e^{-2\left(a s_{i}\right)^{b}}\right\}^{2 \alpha}
\end{array}\right]-\left[\begin{array}{c}
(1+\lambda)\left\{1-e^{-2\left(a s_{i-1}\right)^{b}}\right\}^{\alpha} \\
-\lambda\left\{1-e^{-2\left(a s_{i-1}\right)^{b}}\right\}^{2 \alpha}
\end{array}\right]\right\}}+\sum^{m_{2}} \frac{\mathbf{B}^{(\alpha)}\left(r_{i}\right)}{1-\left[\begin{array}{c}
(1+\lambda)\left\{1-e^{-2\left(a r_{i}\right)^{b}}\right\}^{\alpha} \\
-\lambda\left\{1-e^{-2\left(a r_{i}\right)^{b}}\right\}^{2 \alpha}
\end{array}\right]} \\
& \frac{\partial \ell_{m}(\underline{\Psi})}{\partial a}=\frac{m_{0} b}{a}+(\alpha-1) 2 b a^{b-1} \sum_{i=1}^{m_{0}} \frac{x_{i}^{b} e^{-2\left(a w_{i}\right)^{b}}}{1-e^{-2\left(a w_{i}\right)^{b}}}-2 \alpha \lambda \sum_{i=1}^{m_{0}} \frac{\left[2 b a^{b-1} w_{i}^{b} e^{-2\left(a w_{i}\right)^{b}}\right]\left\{1-e^{-2\left(a w_{i}\right)^{b}}\right\}^{\alpha-1}}{1+\lambda-2 \lambda\left\{1-e^{-2\left(a w_{i}\right)^{b}}\right\}^{\alpha}} \\
& +\sum_{i=1}^{m_{1}} \frac{\left[\mathbf{C}^{(a)}\left(s_{i}\right)-\mathbf{C}^{(a)}\left(s_{i-1}\right)\right]}{\left\{\begin{array}{c}
(1+\lambda)\left\{1-e^{-2\left(a s_{i}\right)^{b}}\right\}^{\alpha} \\
-\lambda\left\{1-e^{-2\left(a s_{i}\right)^{b}}\right\}^{2 \alpha}
\end{array}\right]}+\sum_{i=1}^{m_{2}} \frac{\mathbf{C}^{(a)}\left(r_{i}\right)}{1-\left[\begin{array}{c}
(1+\lambda)\left\{1-e^{-2\left(a r_{i}\right)^{b}}\right\}^{\alpha} \\
-\lambda\left\{1-e^{-2\left(a r_{i}\right)^{b}}\right\}^{2 \alpha}
\end{array}\right]}
\end{aligned}
$$

and

$$
\begin{aligned}
& \frac{\partial \ell_{m}(\underline{\Psi})}{\partial b}=\frac{m_{0}}{b}+n \log a+\sum_{i=1}^{m_{0}} \log \left(x_{i}\right)-2 \sum_{i=1}^{m_{0}} \frac{\log \left(\alpha w_{i}\right)}{\left(\alpha w_{i}\right)^{-b}}+\sum_{i=1}^{m_{0}} \frac{-4 \alpha \lambda\left(a w_{i}\right)^{b} e^{-2\left(a w_{i}\right)^{b}}\left\{1-e^{-2\left(a w_{i}\right)^{b}}\right\}^{\alpha-1}}{1+\lambda-2 \lambda\left\{1-e^{-2\left(a w_{i}\right)^{b}}\right\}^{\alpha}} \\
& +\sum_{i=1}^{m_{1}} \frac{\left[\mathbf{D}^{(b)}\left(s_{i}\right)-\mathbf{D}^{(b)}\left(s_{i-1}\right)\right]}{\left\{\begin{array}{c}
(1+\lambda)\left\{1-e^{-2\left(a s_{i}\right)^{b}}\right\}^{\alpha} \\
-\lambda\left\{1-e^{-2\left(a s_{i}\right)^{b}}\right\}^{2 \alpha}
\end{array}\right]}+\sum_{i=1}^{m_{2}} \frac{\mathbf{D}^{(b)}\left(r_{i}\right)}{1-\left[\begin{array}{c}
(1+\lambda)\left\{1-e^{-2\left(a r_{i}\right)^{b}}\right\}^{\alpha} \\
-\lambda\left\{1-e^{-2\left(a r_{i}\right)^{b}}\right\}^{2 \alpha}
\end{array}\right]},
\end{aligned}
$$

where

$$
\begin{gathered}
\mathbf{A}^{(\lambda)}\left(s_{i}\right)=-\left\{1-e^{-2\left(a s_{i}\right)^{b}}\right\}^{2 \alpha}+\left\{1-e^{-2\left(a s_{i}\right)^{b}}\right\}^{\alpha}, \\
\mathbf{B}^{(\alpha)}\left(s_{i}\right)=\log \left\{1-e^{-2\left(a s_{i}\right)^{b}}\right\}\left\{1-e^{-2\left(a s_{i}\right)^{b}}\right\}^{\alpha}\left(1-2 \lambda\left\{1-e^{-2\left(a s_{i}\right)^{b}}\right\}^{\alpha}+\lambda\right), \\
\mathbf{C}^{(a)}\left(s_{i}\right)=2 \alpha b s_{i}\left(a s_{i}\right)^{b-1} e^{-2\left(a s_{i}\right)^{b}}\left\{1-e^{-2\left(a s_{i}\right)^{b}}\right\}^{\alpha-1}\left(1-2 \lambda\left\{1-e^{-2\left(a s_{i}\right)^{b}}\right\}^{\alpha}+\lambda\right), \\
\mathbf{D}^{(b)}\left(s_{i}\right)=2 \alpha\left(a s_{i}\right)^{b} \log \left(a s_{i}\right) e^{-2\left(a s_{i}\right)^{b}}\left\{1-e^{-2\left(a s_{i}\right)^{b}}\right\}^{\alpha-1}\left(1-2 \lambda\left\{1-e^{-2\left(a s_{i}\right)^{b}}\right\}^{\alpha}+\lambda\right), \\
\mathbf{A}^{(\lambda)}\left(s_{i-1}\right)=\left\{1-e^{-2\left(a s_{i-1}\right)^{b}}\right\}^{\alpha}-\left\{1-e^{-2\left(a s_{i-1}\right)^{b}}\right\}^{2 \alpha},
\end{gathered}
$$




$$
\begin{gathered}
\mathbf{B}^{(\alpha)}\left(s_{i-1}\right)=\log \left\{1-e^{-2\left(a s_{i-1}\right)^{b}}\right\}\left\{1-e^{-2\left(a s_{i-1}\right)^{b}}\right\}^{\alpha}\left(1-2 \lambda\left\{1-e^{-2\left(a s_{i-1}\right)^{b}}\right\}^{\alpha}+\lambda\right), \\
\mathbf{C}^{(a)}\left(s_{i-1}\right)=2 \alpha b s_{i-1}\left(a s_{i-1}\right)^{b-1} e^{-2\left(a s_{i-1}\right)^{b}}\left\{1-e^{-2\left(a s_{i-1}\right)^{b}}\right\}^{\alpha-1}\left(1-2 \lambda\left\{1-e^{-2\left(a s_{i-1}\right)^{b}}\right\}^{\alpha}+\lambda\right), \\
\mathbf{D}^{(b)}\left(s_{i-1}\right)=2 \alpha\left(a s_{i-1}\right)^{b} \log \left(a s_{i-1}\right) e^{-2\left(a s_{i-1}\right)^{b}}\left\{1-e^{-2\left(a s_{i-1}\right)^{b}}\right\}^{\alpha-1}\left(1-2 \lambda\left\{1-e^{-2\left(a s_{i-1}\right)^{b}}\right\}^{\alpha}+\lambda\right), \\
\mathbf{A}^{(\lambda)}\left(r_{i}\right)=\left\{1-e^{-2\left(a r_{i}\right)^{b}}\right\}^{2 \alpha}-\left\{1-e^{-2\left(a r_{i}\right)^{b}}\right\}^{\alpha}, \\
\mathbf{B}^{(\alpha)}\left(r_{i}\right)=\log \left\{1-e^{-2\left(a r_{i}\right)^{b}}\right\}\left\{1-e^{-2\left(a r_{i}\right)^{b}}\right\}^{\alpha}\left(1-2 \lambda\left\{1-e^{-2\left(a r_{i}\right)^{b}}\right\}^{\alpha}+\lambda\right) \\
\mathbf{C}^{(a)}\left(r_{i}\right)=2 \alpha b r_{i}\left(a r_{i}\right)^{b-1} e^{-2\left(a r_{i}\right)^{b}}\left\{1-e^{-2\left(a r_{i}\right)^{b}}\right\}^{\alpha-1}\left(1-2 \lambda\left\{1-e^{-2\left(a r_{i}\right)^{b}}\right\}^{\alpha}+\lambda\right),
\end{gathered}
$$

and

$$
\mathbf{D}^{(b)}\left(r_{i}\right)=2 \alpha\left(a r_{i}\right)^{b} \log \left(a r_{i}\right) e^{-2\left(a r_{i}\right)^{b}}\left\{1-e^{-2\left(a r_{i}\right)^{b}}\right\}^{\alpha-1}\left(1-2 \lambda\left\{1-e^{-2\left(a r_{i}\right)^{b}}\right\}^{\alpha}+\lambda\right) .
$$

